\title{
Molecular Cloning and Characterization of a P38-Like Mitogen-Activated Protein Kinase from Echinococcus granulosus
}

\author{
Guodong Lü1, Jing Li',2, Chuanshan Zhang', Liang Li', Xiaojuan Bi', Chaowang Li', Jinliang Fan', Xiaomei Lu', \\ Dominique A. Vuitton ${ }^{3}$, Hao Wen ${ }^{1, *}$, Renyong Lin ${ }^{1, *}$ \\ 'Xinjiang Key Laboratory of Echinococcosis, Clinical Medical Research Institute, The First Affiliated Hospital of Xinjiang Medical University, Urumqi, \\ Xinjiang, 830054, P. R. China; ${ }^{2}$ College of Basic Medicine, Xinjiang Medical University, Urumqi, Xinjiang, China; ${ }^{3}$ WHO-Collaborating Centre for the \\ Prevention and Treatment of Human Echinococcosis, Department of Parasitology, University of Franche-Comté (EA 3181) and University Hospital, \\ Besançon, France
}

\begin{abstract}
Cystic echinococcosis (CE) treatment urgently requires a novel drug. The p38 mitogen-activated protein kinases (MAPKs) are a family of Ser/Thr protein kinases, but still have to be characterized in Echinococcus granulosus. We identified a 1,107 bp cDNA encoding a 368 amino acid MAPK protein (Egp38) in E. granulosus. Egp38 exhibits 2 distinguishing features of p38-like kinases: a highly conserved T-X-Y motif and an activation loop segment. Structural homology modeling indicated a conserved structure among Egp38, EmMPK2, and $H$. sapiens $\mathrm{p} 38 \mathrm{a}$, implying a common binding mechanism for the ligand domain and downstream signal transduction processing similar to that described for p38a. Egp38 and its phosphorylated form are expressed in the E. granulosus larval stages vesicle and protoscolices during intermediate host infection of an intermediate host. Treatment of in vitro cultivated protoscolices with the p38-MAPK inhibitor ML3403 effectively suppressed Egp38 activity and led to significant protoscolices death within 5 days. Treatment of in vitro-cultivated protoscolices with TGF- $\beta 1$ effectively induced Egp38 phosphorylation. In summary, the MAPK, Egp38, was identified in E. granulosus, as an anti-CE drug target and participates in the interplay between the host and $E$. granulosus via human TGF- $\beta 1$.
\end{abstract}

Key words: Echinococcus granulosus, cystic echinococcosis, protoscolex, p38 MAP kinase

\section{INTRODUCTION}

The p38 mitogen-activated protein kinase (MAPK) is a conserved gene [1-3] whose encoded protein transduces signals from the cell membrane to the nucleus in response to a wide variety of exogenous and endogenous stimuli [4]. Additionally, p38 regulates the expression of many cytokines, transcription factors, and cell surface receptors [5], leading to changes in cell differentiation and apoptotic processes.

As p38 proteins are widely involved in biological processes, their perturbation may lead to diseases. Many chemical compounds possessing p38 inhibitory properties have been identified. In particular, ML3404 and 2 other small molecule inhibi-

- Received 29 March 2016, revised 29 September 2016, accepted 4 October 2016.

*Corresponding authors (renyong_lin@sina.com; dr.wenhao@163.com)

(c) 2016, Korean Society for Parasitology and Tropical Medicine

This is an Open Access article distributed under the terms of the Creative Commons Attribution Non-Commercial License (http://creativecommons.org/licenses/by-nc/4.0) which permits unrestricted non-commercial use, distribution, and reproduction in any

medium, provided the original work is properly cited. tors, SB203580 and AZ10164773, dephosphorylate the active p38 molecule to produce its inactive form. Studies have assessed pharmaceutical drugs specifically targeting p38 to treat or cure certain human diseases, such as pulmonary artery remodeling [6], pancreatic cancer [7], and breast cancer [8]. Furthermore, p38 is not only an important signaling pathway member in the host, but also in invading pathogens.

Cystic echinococcosis (CE) is a cosmopolitan zoonosis caused by $E$. granulosus. It is most prevalent in countries where livestock (the intermediate host) are raised in association with dogs (the definitive host) [9]. The disease not only causes significant economic burdens [10], but also harms human health. At present, albendazole remains the first drug of choice for CE as recommended by WHO [11-13]; however, due to its heavy side effects and low absorption rate, albendazole is not recommended for a long-term hydatid therapy [14-16]. Therefore, identification of new drug targets is urgently required to develop improved therapies to cure CE.

Helminths rely on MAPK pathway signaling to mediate 
their biological processes, and signaling related molecules may be useful drug targets to combat parasitic infections and diseases. The p38 MAPK pathways play important roles in axon regeneration $[17,18]$, and p38 MAPK contributes to ciliary beating in the free-living miracidium stage of the platyhelminth parasite Schistosoma mansoni [19]. According to Patel et al. [20], p38 MAPK pathway activation within the filarial parasite Brugia malayi protects the parasite from destruction by host-produced reactive oxygen species (ROS). Thus, inhibition of this parasite protective pathway may result in therapeutic benefits for the treatment of filariasis by increasing the sensitivity of filarial parasites to ROS and other reactive intermediates. Indeed, Gelmedin et al. [21] identified a p38 homologue in Echinococcus multilocularis that was inhibited at lower doses of SB202190 and ML3403 than the human orthologue Hsp38a; furthermore, Lv et al. [22] confirmed the adequate in vitro scolicidal effects of a specific p38 MAPK inhibitor (SB202190) on E. granulosus. Thus, p38 MAPK may play an important role in E. granulosus and may represent a potential drug target for the treatment of CE. However, the function of the p38 MAPK in E. granulosus, remains uncharacterized, and it is unknown whether other p38 MAPK inhibitors exert on in vitro scolicidal effects on E. granulosus.

\section{MATERIALS AND METHODS}

\section{Animals used in this study}

E. granulosus vesicles and protoscolices were isolated from the livers of sheep naturally-infected with $\mathrm{CE}$, which were obtained from a local commercial slaughterhouse. Two pathogen-free female New Zealand rabbits used for anti-Egp38 antibody preparation were purchased from the animal center of Xinjiang Medical University, housed in cages with a 12-hr light/dark cycle, and provided normal rabbit feed and water. All animals received humane care in compliance with the guidelines of the Medical Research Center, and animal procedures were approved by the Animal Care and User Committee and the Ethical Committee of the First Affiliated Hospital of Xinjiang Medical University, China (20081205-2).

\section{Nucleic acid isolation, Egp38 cloning, and sequencing}

Total RNA was isolated from E. granulosus protoscolices using TRIzol reagent (Invitrogen Co., Beverly, Massachusetts, USA) according to the manufacturer's instructions. The cDNA was then synthesized from the purified RNA according to the instructions included with the reverse transcription kit (Invitrogen) and used as a template to amplify the Egp38 cDNA with the primers p38-5EC (5'-GTGCCCGATGTAAATGAGCG-3') and p38-3EC (5'-CGCGTTGATTGGCGAGTAC-3') [21]. The following thermal cycling conditions were used: 35 cycles of $30 \mathrm{sec}$ at $94^{\circ} \mathrm{C}, 1 \mathrm{~min}$ at $53^{\circ} \mathrm{C}$, and $1 \mathrm{~min}$ at $72^{\circ} \mathrm{C}$. The amplified fragment was cloned into the pMD18-T vector with a TA cloning kit (Takara Co., Dalian, China) and verified by sequencing (Shanghai Sangon Co., Shanghai, China).

\section{Bioinformatics software analysis}

Amino acid sequences of the p38 MAPK homologous genes in E. multilocularis, Homo sapiens, Drosophila melanogaster, Caenorhabditis elegans, and Saccharomyces cerevisiae were downloaded from GenBank (https://www.ncbi.nlm.nih.gov/genbank/) and compared using DNAMAN 5.0. Phylogenetic trees were constructed with the software tool MegAlign for multiple sequence alignment. Catalytic domains were predicted with a simple modular architecture research tool (SMART; http:// smart.embl-heidel-berg.de) [23]. GraphPad Prism software (GraphPad Software) version 5 was used for statistical analyses.

\section{Structure prediction using homology modeling}

The 3 dimensional-structures of the Egp38 proteins were predicted by submitting the amino acid sequences to the SwissModel Automatic Modeling server (http://Swissmodel. expasy.org) [24]. The Egp38 homology model was calculated based on the crystal structure of the Cryptosporidium Ser/Thr protein kinase template (3eb0A). The EmMPK2 homology model was calculated based on the crystal structure of the human tau Ser/Thr protein kinase template (1j1bB). Structural comparisons and figure generation were carried out using DeepView/Swiss-Pdbviewer 4.01 [25].

\section{Quantitative real-time PCR (qRT-PCR) analysis}

Equal amounts of E. granulosus vesicles and protoscolices CDNA were employed for qRT-PCR to determine the expression of Egp38 using the primers p38-5q (5'-ACTCAACTACACCAGCAAGAAG-3') and p38-3q (5'-TCCTACAGGCTCATCACTCTC-3') or elp using the primers elp-5q (5'-CGGGAG GGTAATGACAAG-3') and elp-3q (5'-GGAAGTAACCGTAGTGATGGAG-3'). The following thermal cycling conditions were employed: initiation with a 10 min denaturation at $95^{\circ} \mathrm{C}$, followed by 40 cycles of $30 \mathrm{sec}$ at $94^{\circ} \mathrm{C}$ and $60 \mathrm{sec}$ at $60^{\circ} \mathrm{C}$. The 
relative gene expression of Egp38 was calculated via the $2^{-\Delta \Delta C T}$ method [26]; elp was used as a housekeeping gene [27]. In all cases, each PCR trial was performed in triplicate and repeated at least 3 times.

\section{Heterologous expression in Escherichia coli and purification of recombinant proteins}

Egp38 was expressed in E. coli using the pET-28a system. Briefly, the entire Egp38 open reading frame (ORF) was PCRamplified from the plasmid pMD18-T-Egp38 using the primers p38-F-28a (5'-GGAATTCATGCCCGATGTAAATGA-3') and p38-R-28a (5'-GTCGACCGTTGATTGGCGAGTA-3'), employing the conditions described above, and cloned into the pET28a vector with EcoR I and Sal I, resulting in an N-terminal inframe fusion with a hex-histidine tag. The production of recombinant proteins after induction with $0.1 \mathrm{mM}$ IPTG was carried out according to the pET user manual. The recombinant Egp38 proteins were purified from E. coli lysates under native conditions via affinity chromatography against the hexhistidine tag. After collecting recombinant $E$. coli by centrifugation $\left(6,000 \mathrm{~g}, 20 \mathrm{~min}, 4^{\circ} \mathrm{C}\right)$ from $2 \mathrm{~L}$ of culture, the pellet was resuspended in $20 \mathrm{ml}$ of lysis buffer $(300 \mathrm{mM} \mathrm{NaCl}, 50 \mathrm{mM}$ sodium-phosphate buffer at $\mathrm{pH}$ 8.0, $10 \mathrm{mM}$ imidazole). Cell debris was removed by centrifugation, and the supernatant was transferred to $4 \mathrm{ml}$ of a nickel bead slurry and equilibrated with lysis buffer. After overnight rotation at $4^{\circ} \mathrm{C}$, beads were washed 3 times with $300 \mathrm{ml}$ of washing buffer $(300 \mathrm{mM}$ $\mathrm{NaCl}, 50 \mathrm{mM}$ sodium-phosphate buffer at pH 8.0, $20 \mathrm{mM}$ imidazole). Recombinant protein was then extracted with $6 \mathrm{ml}$ elution buffer I ( $300 \mathrm{mM} \mathrm{NaCl}, 50 \mathrm{mM}$ sodium-phosphate buffer at pH 8.0, $250 \mathrm{mM}$ imidazole) and $6 \mathrm{ml}$ elution buffer II ( $300 \mathrm{mM} \mathrm{NaCl}, 50 \mathrm{mM}$ sodium-phosphate buffer at pH 8.0, $500 \mathrm{mM}$ imidazole). Protein-containing fractions were pooled and dialyzed overnight against PBS at $4^{\circ} \mathrm{C}$.

\section{Antibodies, western blotting analysis, and immunohistochemistry}

To detect Egp38 in Echinococcus larval stages, we generated an anti-Egp38 antibody in rabbits by carrying out 4 rounds of immunization with recombinant Egp38. As the pTGpY phosphorylation motif is highly conserved in Egp38, a commercially available antibody for the phosphorylated (pTGpY) form of p38 MAPK (Promega, Mannheim, Germany) was used to detect phosphorylated Egp38. Rabbit anti- $\beta$-actin (Santa Cruz Biotechnology, Santa Cruz, California, USA) was used to detect $\beta$-actin of $E$. granulosus. For western blotting, vesicles and protoscolices lysates from CE sheep were produced, separated on $12 \%$ acrylamide gels, and electro-transferred to nitrocellulose membranes. Detection was performed with the above-mentioned antibodies according to the manufacturer's instructions, using an anti-rabbit (whole molecule) IgG-peroxidase antibody as the secondary antibody (SigmaAldrich, Taufkirchen, Germany). For immunohistochemistry, vesicles and protoscolices from infected CE sheep were isolated and embedded in paraffin for sectioning and antibody staining. Immunohistochemistry was then performed with the phospho-specific Egp38 antibody (see above) according to a previously established protocol [28].

\section{Drug treatment of protoscolices}

Freshly isolated E. granulosus protoscolices were washed in sterile PBS (Gibco-BRL, Eggenstein, Germany) and incubated in 96-well plates (approximately 400 protoscolices per well) in the presence of culture medium (89\% Dulbecco's minimal essential medium, $10 \%$ fetal calf serum, $1 \%$ penicillin-streptomycin solution). ML3403 was dissolved in dimethylsulphoxide (DMSO). Negative control groups did not contain DMSO or ML3403 (Calbiochem, Gibbstown, New Jersey, USA). The DMSO group which had $2 \mu \mathrm{l}$ of DMSO per well is solvent control. The $7.5 \mu \mathrm{M}, 15 \mu \mathrm{M}, 30 \mu \mathrm{M}$, and $60 \mu \mathrm{M} \mathrm{ML} 3403$ groups contained $2 \mu \mathrm{l}$ of the appropriate concentration of ML3403 per well to achieve the desired final concentrations. At each time point $(0,1,2,3,4$, and 5 days), 3 wells for each group were stained with eosin, and the viability was immediately assessed by counting under a microscope. Red stained protoscolices were dead, and protoscolies lacking color staining were alive. The survival percentage was calculated as the number of live protoscolices divided by the total number of live and dead protoscolices. All experiments were performed independently in quadruplicate.

In addition, freshly prepared protoscolices were treated with $60 \mu \mathrm{M}$ ML3403 for $4 \mathrm{hr}$ in vitro, to detect native or phosphorylated Egp38 by western blotting.

\section{TGF- $\beta 1$ treatment of protoscolices}

Freshly prepared protoscolices were acclimated via cultivation in culture medium (99\% Dulbecco's minimal essential medium, $1 \%$ penicillin-streptomycin solution) for 7 days, then treated with $1 \mathrm{nM}$ TGF- $\beta 1$ (R\&D Systems, Minneapolis, Minneapolis, USA) [29] for $24 \mathrm{hr}$. At each time point (0 hr, 4 
hr, $8 \mathrm{hr}$, and $24 \mathrm{hr}$ ), protoscolices were removed to detect native or phosphorylated Egp38 by western blotting.

\section{RESULTS}

\section{Characteristics of Egp38}

The entire Egp38 ORF sequence comprised 1,107 bp and 368 codons (excluding the TGA stop codon). As shown in Fig. 1, the encoded protein (368 amino acids, $42 \mathrm{kDa}$ ) exhibited significant homology with $\mathrm{p} 38$ proteins in mammals, insects, nematodes, yeasts, and plants. The p38 homologue EmMPK2 from E. multilocularis demonstrated the highest level of homology (98.6\% identity). Lower levels (43.0-61.9\% identity) of homology were shared with p38 homologues from a wide variety of phylogenetically distinct animals, including mammals, insects, and nematodes. Thus, we designated the corresponding $E$. granulosus cDNA and the encoded $42 \mathrm{kDa}$ protein as Egp38.

Based on EmMPK2 sequence analysis [21], the amino acid sequence of Egp38 exhibits many characteristics of p38 homo- logues. A Ser/Thr protein kinase catalytic domain between residues $\mathrm{Tyr}^{25}$ and Phe ${ }^{309}$ of Egp38 was identified with the SMART software package [23]. Many conserved amino acid residues in eukaryotic protein kinases were also observed in EgP38 and at corresponding positions (Fig. 1). Furthermore, Egp38 contains a T-G-Y dual phosphorylation site in the activation loop of the kinase domain.

We investigated the phylogenetic relatedness of p38 homologue sequences. The constructed evolutionary tree revealed the close identity of p38 in E. granulosus and E. multilocularis to orthologs in Schistosoma japonicum and yeast, but divergence from p38 in nematodes, filarial parasites, amphibians, and mammals (Fig. 2).

\section{Homology model of Egp38}

The models for Egp38, EmMPK2, and p38a were very similar (Fig. 3A-C), particularly in terms of the catalytic domain, as illustrated by color-coding. Only minimal differences were observed between the N-terminal regions of Egp38 ( Pro $\left.^{22}-\mathrm{Met}^{110}\right)$

Egp38
EmMPK2
Hsp38a
DmMK14A
CePMK1
ScH0G1
Egp38
EmMPK2
Hsp38a
DmMK14A
CePMK1
ScH0G1
Egp38
EmMPK2
Hsp38a
DmMK14A
CePMK1
ScH0G1
Egp38
EmMPK2
Hsp38a
DmMK14A
CePMK1
ScH0G1

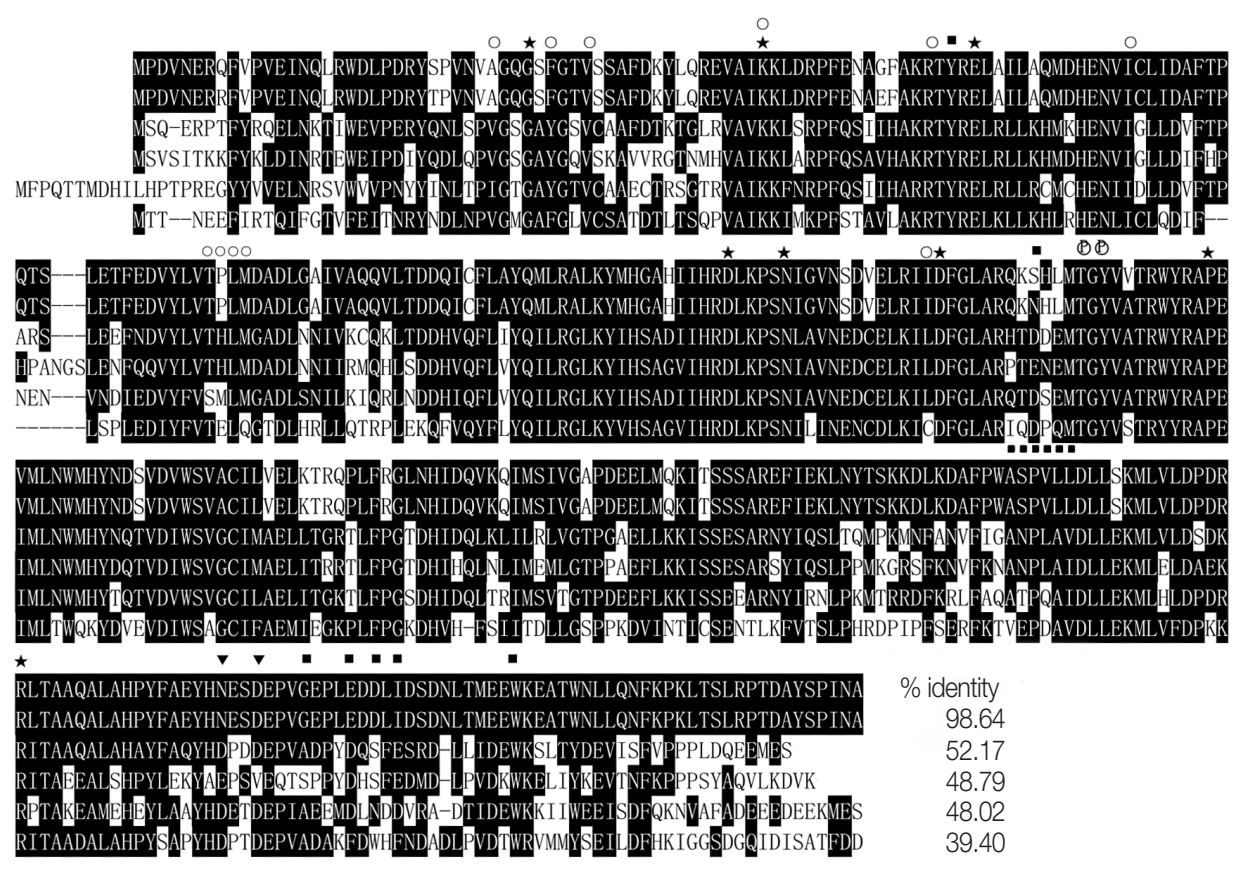

Fig. 1. Alignment of the deduced Egp38 amino acid sequence with other relevant sequences. Amino acid residues that are conserved in at least 2 proteins are indicated in white on a black background. Homologies of the aligned proteins to Egp38 are indicated at the end of the alignment (\% identity). Asterisks denote residues that are highly conserved in eukaryotic protein kinases [37]. Black triangles indicate conserved Asp residues within the docking domain [38]. Thr and Tyr residues of the conserved T-G-Y motif are denoted by ' $\mathrm{B}$ '. Black rectangles indicate residues in the yeast HOG1 that, when mutated, lead to constitutive activation [39]. Circles above the alignment indicate residues in the human p38 MAPK-a that are involved in the binding of pyridinyl imidazole inhibitors [39]. The activation loop is marked by a dotted line below the aligned sequence. Egp38 (ACT21201.1), E. multilocularis p38 MAPK (EmMPK2; CAQ34816.1), H. sapiens p38 MAPK-a (Hsp38a; Q16539), D. melanogaster p38 MAPK (DmMK14A; O62618), C. elegans stress-activated protein kinase PMK-1 (CePMK1; Q17446), and S. cerevisiae HOG-1 (ScHOG1; P32485). 
and EmMPK2 $\left(\mathrm{Asp}^{23}-\mathrm{Met}^{110}\right)$. In contrast, the N- and C-terminal regions of $\mathrm{p} 38 \mathrm{a}$ (Fig. 3C) were very different compared to Egp38 and EmMPK2; in particular, p38a included 2 longer helices (colored sky blue and red).

\section{Egp38 expression during the larval stages of E. granulosus}

As shown in Fig. 4, there were no significant differences between the vesicles and protoscolices of E. granulosus, indicating the constitutive expression of Egp38 cDNA, during the larval stages. An anti-Egp38 antibody and an antibody for the phosphorylated (pTGpY) form of p38 MAPK were employed to detect Egp38 and its active form, respectively. Egp38 and its active form were both identified in Echinococcus vesicles and protoscolices lysates, and the proteins migrated at approximately $45 \mathrm{kDa}$.

\section{Localization of Egp38}

As shown in Fig. 6A and D, both the germinal layer of the vesicle and the defined structures of the protoscolex reacted

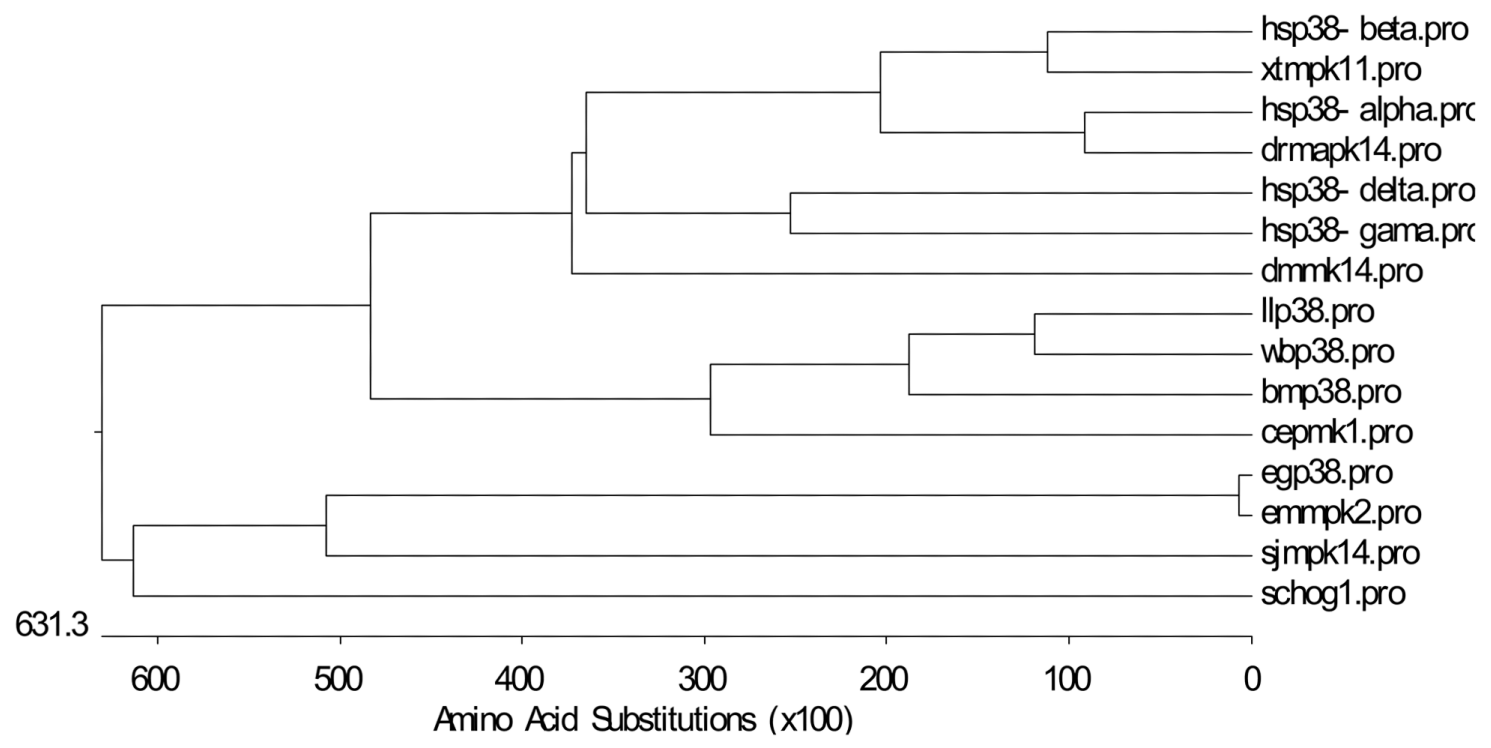

Fig. 2. Phylogenetic tree of p38 in E. granulosus and other relevant species. E. granulosus p38 MAPK (egp38.pro; ACT21201.1), E. multilocularis p38 MAPK (emmpk2.pro; CAQ34816.1), H. sapiens p38 MAPK-a (hsp38-alpha.pro; Q16539), H. sapiens p38 MAPK- $\beta$ (hsp38-beta.pro; Q15759), H. sapiens p38 MAPK-ү (hsp38-gama.pro; P53778), H. sapiens p38 MAPK-ס (hsp38-delta.pro; O15264), C. elegans stress-activated protein kinase PMK-1 (cepmk1.pro; Q17446), Danio rerio Mapk14b protein (drmapk14.pro; AAH63937.1), Xenopus tropicalis MAPK 11 (xtmpk11.pro; NP_001098754.1), S. japonicum MAPK14a (sjmpk14; CAX72870), Brugia malayi p38 map kinase family protein 2 isoform b (bmp38.pro; A8PQS0), Loa loa CMGC/MAPK/p38 protein kinase (llp38.pro; XP_003141640), Wuchereria bancrofti CMGC/MAPK/P38 protein kinase (wbp38.pro; WUBG14523.1 of Broad Institute Filarial Database), D. melanogaster p38 MAPK (dmmk14.pro; O62618), and yeast HOG-1 (schog1.pro; P32485).

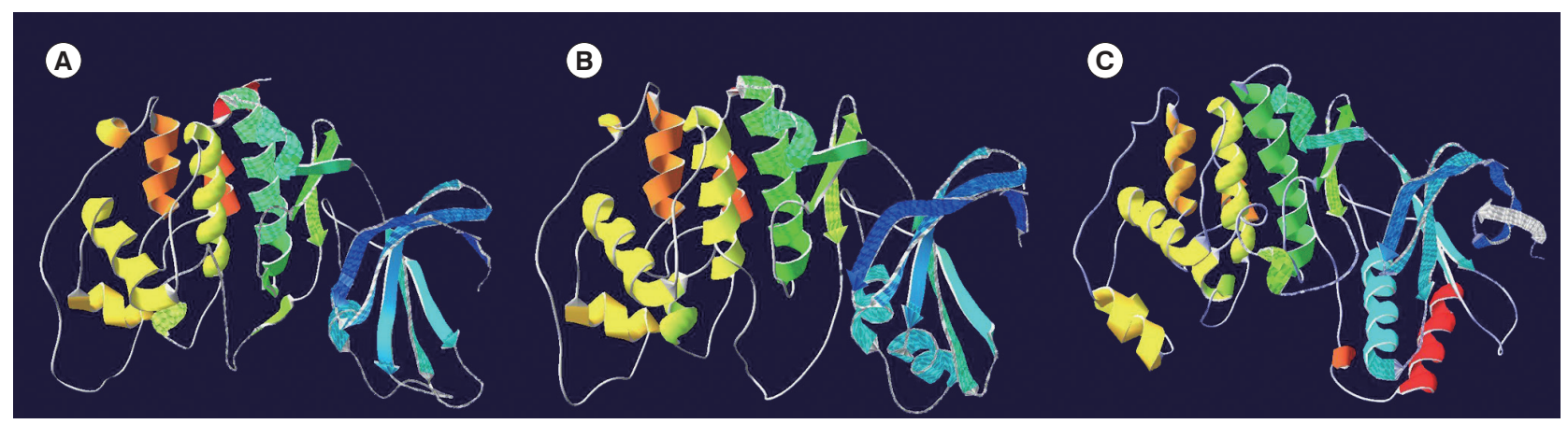

Fig. 3. Molecular models of the tertiary structure. (A) Surface rendering of Egp38. (B) Surface rendering of EmMPK2. (C) Surface rendering of Hsp38a. Individual sub-domains (helix and strand) are colored in the following order: gray, deep blue, sky blue, green, kelly green, yellow, khaki, orange, and red. 
with rabbit-anti Egp38 immune sera, which detected total Egp38. In the vesicles, strong staining was observed in the cytoplasm and nuclei of the germinal layer cells. In the protoscolices, staining was present in the tissue surrounding the rostellum and in the suckers. As shown in Fig. 6B and E, localization of phosphorylated Egp38 was similar to that of the total Egp38 in the germinal layer of the vesicle and protoscolex. However, phosphorylated Egp38 was localized in the cytoplasm and nuclei of certain germinal layer cells in the vesicles.

Taken together, the western blotting and immunohisto-

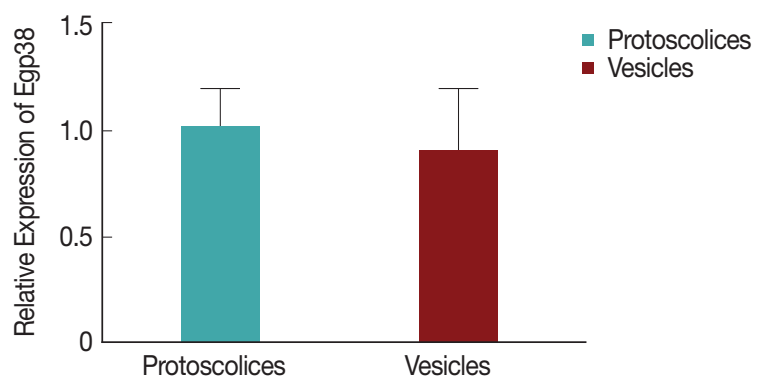

Fig. 4. Egp38 mRNA levels in larval stages. chemistry findings from parasite lysates indicated that Egp38 was constitutively present throughout the Echinococcus larval development stages.

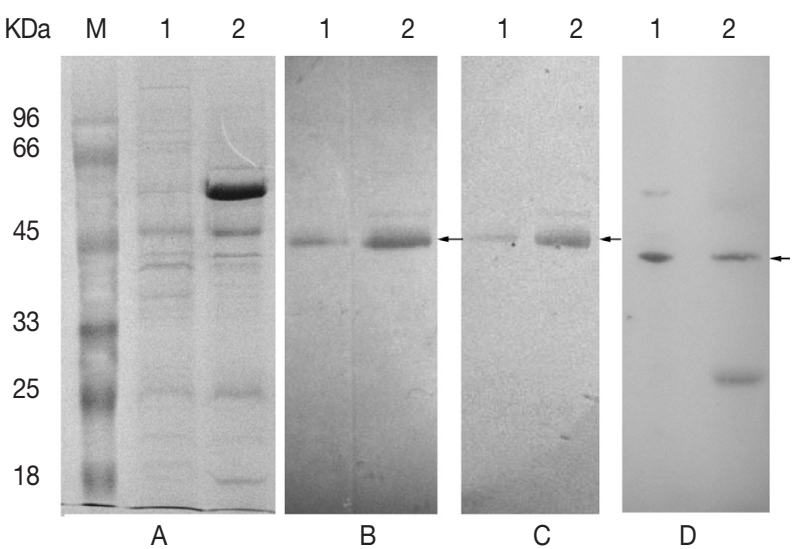

Fig. 5. Egp38 antibody reactivities. (A) Total proteins were separated by SDS-PAGE. (B) The Egp38 protein was identified with an anti-p38 antibody. (C) The Egp38 protein was identified with an anti-phospho-p38 (pTGpY) antibody. (D) $\beta$-actin protein of $E$. granulosus was identified with an anti- $\beta$-actin antibody. Lane $1, E$. granulosus protoscolices lysates; lane $2, E$. granulosus vesicle lysates. Arrows indicate the interest proteins.

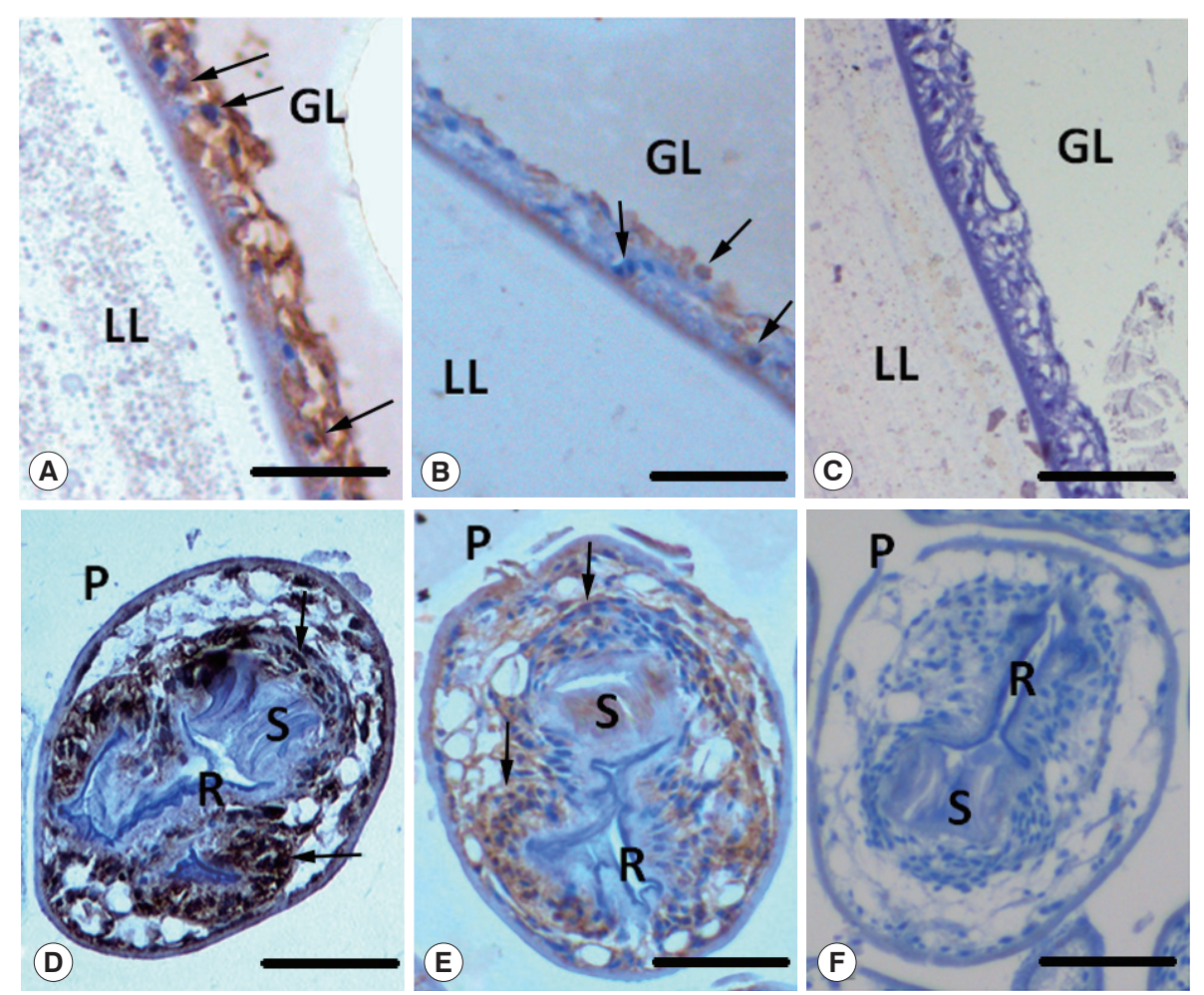

Fig. 6. Immunohistochemical detection of Egp38 in E. granulosus larval stages. Paraffin sections of protoscolices and vesicles isolated from CE sheep were probed with rabbit anti-Egp38 (A, D) and anti-phospho-p38 primary antibodies (B, E) and developed with a peroxidase-coupled goat anti-rabbit lgG secondary antibody or with secondary antibody alone (C, F). The following structures are indicated: GL, germinal layer; LL, laminated layer; P, protoscolex; R, rostellum; S, sucker. Arrows indicate the locations of Egp38 or the active form of Egp38. Scale bar $=50 \mu m$. 
A

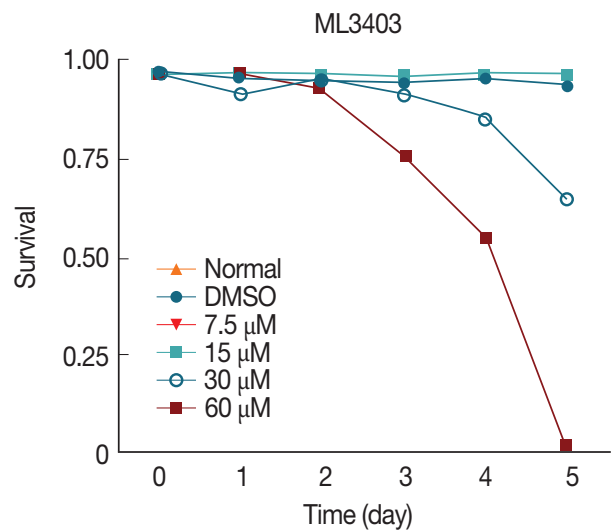

B

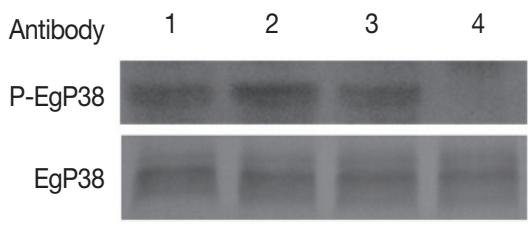

Fig. 7. Effects of the p38 MAPK inhibitor ML3403 on the viability of cultivated protoscolices in vitro. (A) Survival rates of protoscolices after 5 days of treatment with different concentrations of ML3403. (B) Egp38 activity in inhibitor-treated protoscolices. Protoscolices were incubated with the inhibitor ML3403 (60 $\mu \mathrm{M}$ ) for $4 \mathrm{hr}$ (lane 4). Lysates were examined by western blotting using antibodies against phosphorylated p38 MAPK (P-Egp38) as well as non-phosphorylated (Egp38). Lane 1, protoscolices prior to treatment. Lane 2, untreated protoscolices grown for $4 \mathrm{hr}$. Lane 3, DMSO treatment for $4 \mathrm{hr}$. Lane 4, ML3403 treatment for $4 \mathrm{hr}$.

A

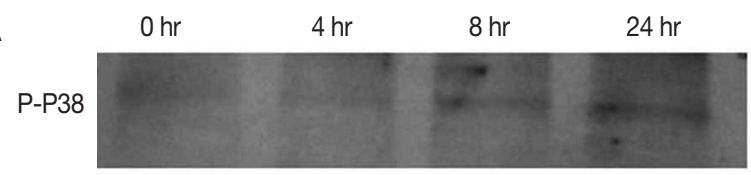

P38

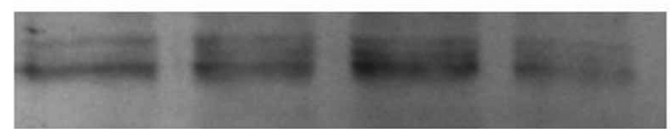

B

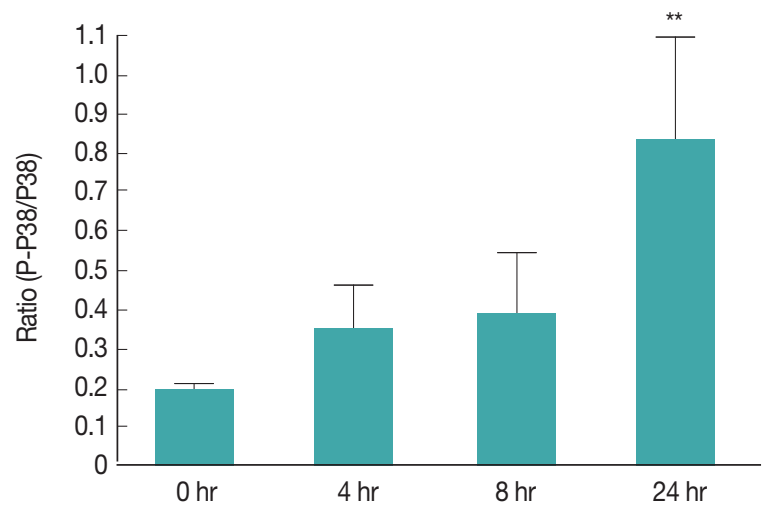

Fig. 8. EgP38 phosphorylation in protoscolices cultivated with TGF- $\beta 1$ in vitro. (A) Time-dependent phosphorylation of EgP38 following TGF- $\beta 1$ treatment of protoscolices; immunoblotting was performed with either antibody as shown in Fig. 7. (B) Quantification by densitometry $\left(n=4 \pm S D\right.$; ${ }^{*} P<0.01$ compared the with 0 hr group).

\section{Effects of p38 MAPK inhibitors on cultivated protoscolices in vitro}

To test the effects of p38 MAPK inhibitors on E. granulosus larvae, we employed ML3403 and examined the phosphorylation status of Egp38. After treatment with $60 \mu \mathrm{M}$ ML3403 for
$4 \mathrm{hr}$, the phospho-Egp38 band nearly disappeared, while the phospho-Egp38 band in the control group, with or without DMSO, was clearly present (Fig. 7B); thus, the p38 MAPK inhibitor effectively blocked Egp38 phosphorylation activity in the parasite.

On the 3rd day, the viability of the protoscolices treated with 30 and $60 \mu \mathrm{M}$ ML3404 (Fig. 7A) began to decline. On the 5th day, the protoscolices viability of the $60 \mu \mathrm{M}$ ML3404 treatment group declined to only $7.3 \%$ of the total (Fig. 7A). However, protoscolices survival in the normal group, the DMSO group, the $7.5 \mu \mathrm{M}$ ML3404 group and the $15 \mu \mathrm{M}$ ML3404 group remained unaffected (Fig. 7A, Supplementary Fig. S1).

\section{Effects of human TGF- $\beta 1$ on cultivated protoscolices in vitro}

To investigate whether human TGF- $\beta 1$ exerts effects via Egp38, we treated E. granulosus protoscolices with human TGF$\beta 1$ for $24 \mathrm{hr}$ in vitro. Human TGF- $\beta 1$ activated the phosphorylation of Egp38 over time. At $24 \mathrm{hr}$, the phosphorylation levels of Egp38 were increased by approximately 4-folds (Fig. 8).

\section{DISCUSSION}

The p38 kinase homologues have been identified in many helminths, such as E. multilocularis, S. japonicum, S. mansoni, and B. malayi, and play crucial roles in supporting parasite survival. Our study yielded evidence supporting the existence of a p38 homologue in E. granulosus. The mRNA and the active protein form of Egp38 were expressed in the germinal layers of 
vesicles and protoscolices. The active form of Egp38 was expressed in both the vesicles and protoscolices, but not in the accompanying cystic fluid (data not shown). This intracellularrestricted expression pattern indicates that Egp38 is a bioactive molecule during the larval stages of $E$. granulosus but does not interact with the host directly because it is not secreted by $E$. granulosus cells. Furthermore, the active form of Egp38 was observed in the cytoplasm and nuclei of the vesicle germinal layer and protoscolices, which agrees with the predominant localization of phosphorylated forms of p38-like kinases in animal cells [31]. Thus, the function of Egp38 is likely similar to that of other p38 homologous genes and transduces extracellular signaling to the nucleus.

In many parasites, the p38 MAP homologue has been identified as a good candidate for the development of drugs to combat parasitic diseases [20,21]. According to Lv H et al. [22], an inhibitor of Egp38, SB202190, exhibits parasiticidal activity in vitro; however, it is unclear whether SB202190 kills the larvae by inhibiting the activity of Egp38 or if Egp38 is an antiCE drug target. In this work, Egp38 influenced the survival of E. granulosus larval stages. Inhibition of the active form of Egp38 in protoscolices, via treatment with the p38-specific inhibitor ML3403 resulted in cell death; thus, Egp38 is important for protoscolex survival. Egp38 may be a viable drug target to cure CE.

The p38 MAPKs are a class of mitogen-activated protein kinases that respond to stress stimuli, such as cytokines (TGF- $\beta 1$ ) [32], and Egp38 contains typically characteristic domains of p38 homologues. TGF- $\beta 1$, an important cytokine that regulates Smad and p38 signaling pathways in mammals, is highly expressed in the pericystic layer of the echinococcus vesicle cyst [33], and in sera of CE patients [34] and mice infected with $E$. granulosus [35]. Human TGF- $\beta 1$ activated Egp38, indicating the ability of the parasite to respond to host TGF- $\beta 1$ and control its parasitism by activating the Egp38 signaling pathway. According to Zhang et al. [27], human TGF- $\beta 1$ activates Smad signaling pathways in E. granulosus. Therefore, human TGF- $\beta 1$ appears to exert multiple functions on $E$. granulosus by regulating the Smad and Egp38 signaling pathways.

EgMKK1 in E. granulosus, whose sequence shares high identity with EmMKK1 in E. multilocularis [36], closely resembles members of the MKK3/6-MAPKK family (data not shown). MKK3/6-MAPKK proteins are upstream signaling molecules of p38 MAPK. Thus far, Egp38 and EgMKK1 are the only 2 members of the p38 MAPK pathway identified in E. granulosus, but there are certainly many other related signaling molecules belonging to this pathway that, when identified, will provide significant insights into the precise mechanisms employed by Egp38.

Collectively, the newly discovered Egp38 MAP kinase will be useful for exploring the molecular mechanisms underlying the interplay between host TGF- $\beta 1$ and E. granulosus and in the development of new anti-parasite drugs to cure CE.

\section{ACKNOWLEDGMENTS}

This work was funded by grants from the National Natural Science Foundation of China (U1303222, 81360251, and 81260252) and the State Key Laboratory Incubation Base of Xinjiang Major Diseases Research Fund, China (SKLIB-XJMDR-2012-2).

\section{CONFLICT OF INTEREST}

We have no conflicts of interest related to this work.

\section{REFERENCES}

1. Posas F, Wurgler-Murphy SM, Maeda T, Witten EA, Thai TC, Saito H. Yeast HOG1 MAP kinase cascade is regulated by a multistep phosphorelay mechanism in the SLN1-YPD1-SSK1 "twocomponent" osmosensor. Cell 1996; 86: 865-875.

2. Wang XS, Diener K, Manthey CL, Wang S, Rosenzweig B, Bray J, Delaney J, Cole CN, Chan-Hui PY, Mantlo N, Lichenstein HS, Zukowski M, Yao Z. Molecular cloning and characterization of a novel p38 mitogen-activated protein kinase. J Biol Chem 1997; 272: 23668-23674.

3. Berman K, McKay J, Avery L, Cobb M. Isolation and characterization of pmk-(1-3): three p38 homologs in Caenorhabditis elegans. Mol Cell Biol Res Commun 2001; 4: 337-344.

4. Raingeaud J, Gupta S, Rogers JS, Dickens M, Han J, Ulevitch RJ, Davis RJ. Pro-inflammatory cytokines and environmental stress cause p38 mitogen-activated protein kinase activation by dual phosphorylation on tyrosine and threonine. J Biol Chem 1995; 270: 7420-7426.

5. Ono K, Han J. The p38 signal transduction pathway: activation and function. Cell Signal 2000; 12: 1-13.

6. Zhao G, Seng J, Beagle J, Syrkina O, Hales CA. Heparin reduces overcirculation-induced pulmonary artery remodeling through p38 MAPK in piglet. Ann Thorac Surg 2015; 99: 1677-1684.

7. Taniuchi K, Furihata M, Hanazaki K, Iwasaki S, Tanaka K, Shimizu T, Saito M, Saibara T. Peroxiredoxin 1 promotes pancreatic cancer cell invasion by modulating p38 MAPK activity. Pancreas 
2015; 44: 331-340.

8. Wang XF, Zhou QM, Lu YY, Zhang H, Huang S, Su SB. Glycyrrhetinic acid potently suppresses breast cancer invasion and metastasis by impairing the p38 MAPK-AP1 signaling axis. Expert Opin Ther Targets 2015; 19: 577-587.

9. Eckert J, Deplazes P. Biological, epidemiological, and clinical aspects of echinococcosis, a zoonosis of increasing concern. Clin Microbiol Rev 2004; 17: 107-135.

10. Budke CM, Jiamin Q, Qian W, Torgerson PR. Economic effects of echinococcosis in a disease-endemic region of the Tibetan Plateau. Am J Trop Med Hyg 2005; 73: 2-10.

11. Arif SH, Shams-Ul-Bari, Wani NA, Zargar SA, Wani MA, Tabassum R, Hussain Z, Baba AA, Lone RA. Albendazole as an adjuvant to the standard surgical management of hydatid cyst liver. Int J Surg 2008; 6: 448-451.

12. Jamshidi M, Mohraz M, Zangeneh M, Jamshidi A. The effect of combination therapy with albendazole and praziquantel on hydatid cyst treatment. Parasitol Res 2008; 103: 195-199.

13. Bildik N, Cevik A, Altintas M, Ekinci H, Canberk M, Gulmen M. Efficacy of preoperative albendazole use according to months in hydatid cyst of the liver. J Clin Gastroenterol 2007; 41: 312-316.

14. Hemphill A, Spicher M, Stadelmann B, Mueller J, Naguleswaran A, Gottstein B, Walker M. Innovative chemotherapeutical treatment options for alveolar and cystic echinococcosis. Parasitology 2007; 134: 1657-1670.

15. Kern P. Medical treatment of echinococcosis under the guidance of Good Clinical Practice (GCP/ICH). Parasitol Int 2006; 55 Suppl: S273-S282.

16. Reuter S, Jensen B, Buttenschoen K, Kratzer W, Kern P. Benzimidazoles in the treatment of alveolar echinococcosis: a comparative study and review of the literature. J Antimicrob Chemother 2000; 46: 451-456.

17. Nix P, Hisamoto N, Matsumoto K, Bastiani M. Axon regeneration requires coordinate activation of p38 and JNK MAPK pathways. Proc Natl Acad Sci USA 2011; 108: 10738-10743.

18. Li C, Hisamoto N, Matsumoto K. Axon regeneration is regulated by Ets-C/EBP transcription complexes generated by activation of the cAMP/Ca ${ }^{2+}$ signaling pathways. PLos Genet 2015; 11: e1005603.

19. Ressurreicao M, Rollinson D, Emery AM, Walker AJ. A role for p38 MAPK in the regulation of ciliary motion in a eukaryote. BMC Cell Biol 2011; 12: 6 .

20. Patel A, Chojnowski AN, Gaskill K, De Martini W, Goldberg RL, Siekierka JJ. The role of a Brugia malayi p38 MAP kinase ortholog (Bm-MPK1) in parasite anti-oxidative stress responses. Mol Biochem Parasitol 2011; 176: 90-97.

21. Gelmedin V, Caballero-Gamiz R, Brehm K. Characterization and inhibition of a p38-like mitogen-activated protein kinase (MAPK) from Echinococcus multilocularis: antiparasitic activities of p38 MAPK inhibitors. Biochem Pharmacol 2008; 76: 1068-1081.

22. Lv H, Li S, Zhang J, Liang W, Mu X, Jiang Y. In vitro effects of SB202190 on Echinococcus granulosus. Korean J Parasitol 2013; 51: 255-258.

23. Schultz J, Milpetz F, Bork P, Ponting CP. SMART, a simple modu- lar architecture research tool: Identification of signaling domains. Proc Natl Acad Sci USA 1998; 95: 5857-5864.

24. Bordoli L, Kiefer F, Arnold K, Benkert P, Battey J, Schwede T. Protein structure homology modeling using SWISS-MODEL workspace. Nat Protoc 2009; 4: 1-13.

25. Guex N, Peitsch MC, Schwede T. Automated comparative protein structure modeling with SWISS-MODEL and Swiss-PdbViewer: a historical perspective. Electrophoresis 2009; 30 Suppl 1: S162-S173.

26. von Schnakenburg C, Strehlau J, Ehrich JH, Melk A. Quantitative gene expression of TGF- $\beta 1$, IL-10, TNF- $\alpha$ and Fas Ligand in renal cortex and medulla. Nephrol Dial Transplant 2002; 17: 573579.

27. Zhang C, Wang L, Wang H, Pu H, Yang L, Li J, Wang J, Lü G, Lu X, Zhang W, Vuitton DA, Wen H, Lin R. Identification and characterization of functional Smad8 and Smad4 homologues from Echinococcus granulosus. Parasitol Res 2014; 113: 3745-3757.

28. Spiliotis M, Konrad C, Gelmedin V, Tappe D, Bruckner S, Mosch HU, Brehm K. Characterisation of EmMPK1, an ERK-like MAP kinase from Echinococcus multilocularis which is activated in response to human epidermal growth factor. Int J Parasitol 2006; 36: 1097-1112.

29. Oliveira KC, Carvalho ML, Verjovski-Almeida S, LoVerde PT. Effect of human TGF- $\beta$ on the gene expression profile of Schistosoma mansoni adult worms. Mol Biochem Parasitol 2012; 183: 132-139.

30. Zhang CC, Shapiro DJ. Activation of the p38 mitogen-activated protein kinase pathway by estrogen or by 4-hydroxytamoxifen is coupled to estrogen receptor-induced apoptosis. J Biol Chem 2000; 275: 479-486.

31. Pomérance M, Quillard J, Chantoux F, Young J, Blondeau JP. High-level expression, activation, and subcellular localization of p38-MAP kinase in thyroid neoplasms. J Pathol 2006; 209: 298306.

32. Tan Y, Xu Q, Li Y, Mao X, Zhang K. Crosstalk between the p38 and TGF- $\beta$ signaling pathways through T $\beta$ RI, T $\beta$ RII and Smad3 expression in plancental choriocarcinoma JEG-3 cells. Oncol Lett 2014; 8: 1307-1311.

33. Jiang T, Niu J, Peng X, Zhang S, Wu X, Sun H. Expression and significance of CTGF and TGF- $\beta 1$ in the pericystic layers of hepatic hydatid cyst. J Shihezi Univ (Natural Science) 2007: 323326.

34. Tuxun T, Wang JH, Lin RY, Shan JY, Tai QW, Li T, Zhang JH, Zhao JM, Wen H. Th17/Treg imbalance in patients with liver cystic echinococcosis. Parasite Immunol 2012; 34: 520-527.

35. Haniloo A, Ghasemi F, Shikhi A, Ghavami M. Immunoregulatory cytokine (TGF-ß and IL-10) responses in mice inoculated with protoscoleces and major hydatid fluid antigens of cystic echinococcosis. Iran J Parasitol 2008; 3: 18-23.

36. Gelmedin V, Spiliotis M, Brehm K. Molecular characterisation of MEK1/2- and MKK3/6-like mitogen-activated protein kinase kinases (МАРКK) from the fox tapeworm Echinococcus multilocularis. Int J Parasitol 2010; 40: 555-567. 
37. Hanks SK, Hunter T. Protein kinases 6. The eukaryotic protein kinase superfamily: kinase (catalytic) domain structure and classification. FASEB J 1995; 9: 576-596.
38. Tanoue T, Nishida E. Docking interactions in the mitogen-activated protein kinase cascades. Pharmacol Ther 2002; 93: 193202.

39. Bell M, Capone R, Pashtan I, Levitzki A, Engelberg D. Isolation of hyperactive mutants of the MAPK p38/Hog1 that are independent of MAPK kinase activation. J Biol Chem 2001; 276: 25351-25358. 


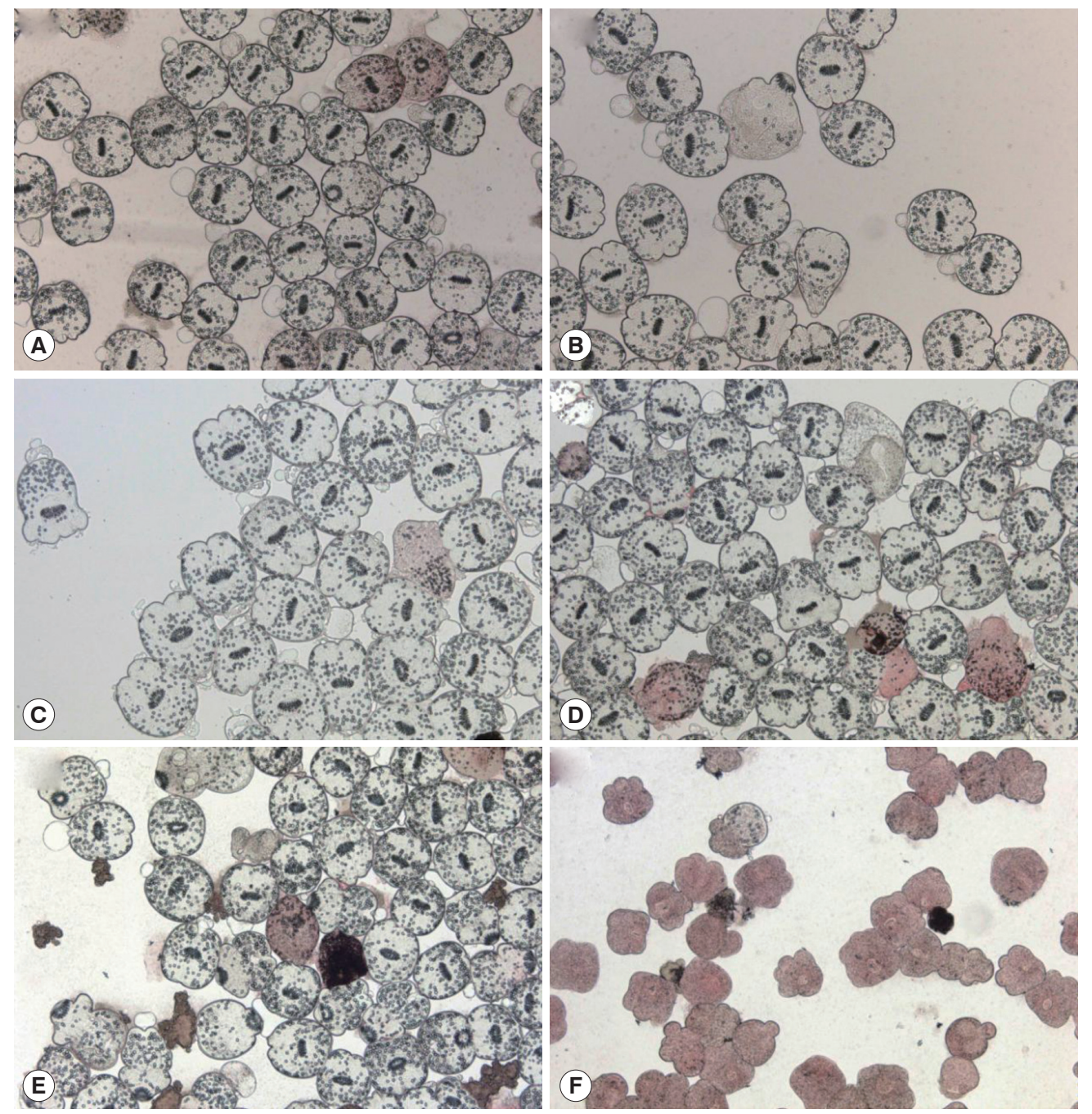

Fig. S1. Protoscolices of $E$. granulosus treated with drugs in vitro. (A) Protoscolices after 5 days of cultivation under normal (untreated)

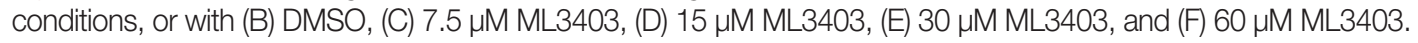


